\title{
7. COMMISSION DE L'ASTRONOMIE DYNAMIQUE ET DES TABLES ASTRONOMIQUES
}

\section{Président: M. Andoyer, Professeur d'Astronomie, Faculté des Sciences de l'Université de Paris.}

Membres: MM. Armellini, Banachiewicz, E. W. Brown, Cowell, Fotheringham, Heinrich, Leuschner, Merlin, Moulton, Nörlund, Sampson, Smart, Strömgren, Woltjer.

Aucune proposition spéciale n'est soumise aux délibérations de la Commission par ses membres. Mais il convient de signaler l'importance toujours plus grande que prend la question de la non-uniformité de la rotation de la terre, intimement liée d'ailleurs à celle des divergences que l'on constate entre la théorie et l'observation de la Lune, du Soleil et des planètes. Les travaux récents de MM. E. W. Brown et J. K. Fotheringham sur ces sujets sont particulièrement importants: sans en rappeler ici le détail, j'exprimerai simplement le vœu qu'ils soient l'objet d'une discussion approfondie à notre prochaine réunion.

Mes recherches sur la théorie analytique du mouvement de la Lune sont maintenant terminées. On trouvera les expressions analytiques complètes des Six Coordonnées de la Lune, tant polaires que rectangulaires, avec une approximation au moins égale à celle de Delaunay, dans les tomes 58 et 59 des Mémoires de l'Académie des Sciences de Paris.

Il convient encore de signaler un article de M. W. M. Smart (M.N., R.A.S., 87) sur les équations de la mécanique céleste et une note de $M$. Merlin sur la Distribution des vitesses et des densités dans un fluide en rotation (C.R. 185).

Je regrette de ne pouvoir donner ici des détails sur les travaux très intéressants de MM. E. Strömgren et W. Heinrich dont la notice m'est parvenue trop tard.

\author{
H. ANDOyer \\ Président de la Commission
}

décembre 27, 1927

\section{Appendix}

\section{CORRECTIONS A APPLIQUER AU TEMPS ASTRONOMIQUE POUR LE CHANGER EN TEMPS NEWTONIEN OU UNIFORME}

\section{Par W. DE Sirter}

Le temps astronomique est le temps mesuré par la rotation de la terre, et qui est employé comme argument dans nos éphémérides et tables astronomiques.

Le tèmps Newtonien, ou uniforme, est la variable indépendante des équations de la dynamique, auquel les moyens mouvements sidéraux des corps célestes sont proportionnels.

D'après les hypothèses et les formules de $B . A . N$. III, No. 124 et 127 la différence entre ces deux temps est causée par la manque d'uniformité de la rotation de la terre. Cette déviation de l'uniformité est composée de deux parties qui sont gouvernées par des lois différentes.

Dans les formules suivantes $T$ est le temps mesuré en siècles à partir de I900.0, et

$$
S=T^{2}+\mathrm{I} \cdot 33 T-0 \cdot 26,
$$

de manière que $S=0$ pour I750.0 et Igr $7 \cdot I$. 
$A$. Changements discontinus du moment d'inertie $C$ de la terre et, par conséquent, de la vitesse de rotation $\omega$.

La différence $\Delta t=$ temps Newtonien - temps astronomique, produite par cette cause est de la forme

$$
(\Delta t)_{A}=a+b T \text {. }
$$

La valeur de $b$ change discontinument quand la valeur de $C$ change, et on a

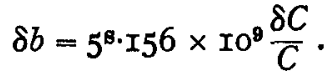

Les constantes $a$ doivent être choisies de telle manière que pour les époques de discontinuité les valeurs de $(\Delta t)_{A}$ calculées par les deux formules avant et

\begin{tabular}{|c|c|}
\hline $\begin{array}{l}\text { Epoque de } \\
\text { discontinuité }\end{array}$ & s $\quad(\Delta t)_{A}$ \\
\hline $\begin{array}{l}1664 \cdot 0 \\
1755 \cdot 3 \\
1786 \cdot 2 \\
1864 \cdot 4 \\
1876 \cdot 15 \\
1896 \cdot 7 \\
1918 \cdot 65\end{array}$ & $\begin{array}{l}-27.8 \\
-0.5+48.7(T+1.80) \\
+21.4+32.1(T+1.30) \\
+13.9-33.0(T+0.75) \\
=6.5-130.1(T+0.30) \\
-20.4-67.6(T+0.15) \\
-23.7+55.8(T-0.05) \\
-17.1-67.6(T-0.20)\end{array}$ \\
\hline
\end{tabular}
après le changement de $b$ soient les mêmes. Les formules adoptées sont

$B$. Retardement de la rotation de la terre par la friction des marées. La partie de $\Delta t$ produite par cette cause est

$$
(\Delta t)_{B}=a^{\prime}+b^{\prime} T+c^{\prime} T^{2} .
$$

On suppose que la constante $c^{\prime}$ peut changer discontinument. Les constantes $a^{\prime}$ et $b^{\prime}$ doivent être choisies de manière que les valeurs de $(\Delta t)_{B}$ et $\operatorname{de} d(\Delta t)_{B} / d t$ soient les mêmes d'après les deux formules avant et après le changement de $c^{\prime}$. Les formules adoptées sont:

$$
\begin{array}{cc}
\begin{array}{c}
\text { Epoque de } \\
\text { discontinuite }
\end{array} & (\Delta t)_{B} \\
1742 \cdot 8 & +22 \cdot 7(T+1 \cdot 709)+43 \cdot 7 S \\
1869 \cdot 0 & -14 \cdot 4(T+1 \cdot 182)+23 \cdot 3 S \\
& -46.9(T+0.017)+69 \cdot 2 S
\end{array}
$$

Les accélérations séculaires des longitudes des corps célestes sont proportionnelles à une valeur moyenne de $(\Delta t)_{B}$, pour laquelle nous adoptons

Soit encore*:

$$
S^{\prime}=40^{8 \cdot 2} S \text {. }
$$

$$
\begin{aligned}
(\Delta t)^{\prime}{ }_{B} & =(\Delta t)_{B}-S^{\prime} \\
\Delta_{1} t & =(\Delta t)_{A}+(\Delta t)^{\prime}{ }_{B} \\
M & =(\Delta t)_{A}+0 \cdot 229(\Delta t)^{\prime}{ }_{B}^{\prime} \\
\Delta t & =S^{\prime}+\Delta_{1} t=(\Delta t)_{A}+(\Delta t)_{B} .
\end{aligned}
$$

Alors l'équation séculaire de la longitude d'un corps céleste autre que la lune, dont le moyen mouvement en secondes d'arc par jour est $n$, est

$$
\delta_{g} \lambda=+\frac{n}{86400} S^{\prime}
$$

* Pour les deux coefficients $Q_{s}$ et $Q$ définis dans B.A.N. III, 124, on a adopté:

$$
Q_{s}=\mathrm{I} / 0 \cdot 229=4 \cdot 37, \quad Q=\text { valeur moyenne de } \Delta_{1} t / M=\mathrm{I} \cdot 246 .
$$


Pour la lune on a, au contraire,

$$
\delta_{8} L=\frac{0.229 n^{\prime}}{86400} S^{\prime}=0^{\prime \prime} \cdot \text { I257 } S^{\prime}
$$

Les fuctuations dans les longitudes des corps célestes, autres que la lune, sont

et dans la longitude de la lune

$$
\delta_{1} \lambda=+\frac{n}{86400} \Delta_{1} t
$$

$$
\delta_{1} L=+\frac{n^{\prime}}{86400} M .
$$

Aux longitudes des corps célestes prises dans les tables astronomiques on doit par conséquent appliquer les corrections

$$
\begin{aligned}
& \delta \lambda=\delta_{8} \lambda+\delta_{1} \lambda=\frac{n}{86400} \Delta t, \\
& \delta L=\delta_{8} L+\delta_{1} L .
\end{aligned}
$$

Ces corrections entraînent des corrections aux longitudes à l'époque et aux moyens mouvements. En outre, dans le cas de la lune, il faut écarter le terme empirique, qui est contenu dans les tables. Ainsi à la longitude de la lune prise dans les tables de Brown il faut appliquer

\begin{tabular}{|c|c|c|c|c|}
\hline$t$ & $\begin{array}{c}S^{\prime} \\
\mathbf{s}\end{array}$ & $\begin{array}{c}\Delta_{1} t \\
\mathrm{~S}\end{array}$ & $\begin{array}{c}M \\
\mathrm{~s}\end{array}$ & $\Delta t$ \\
\hline 1640 & $+122 \cdot 6$ & $-37 \cdot 6$ & $-30.0^{\circ}$ & $\begin{array}{r}s \\
+85.0\end{array}$ \\
\hline 50 & $\begin{array}{r}+107.4 \\
\end{array}$ & $\begin{array}{r}-36.6 \\
-\end{array}$ & $\begin{array}{l}-29.8 \\
-28\end{array}$ & $\begin{array}{r}+ \\
+70.8 \\
\end{array}$ \\
\hline 60 & $\begin{array}{r}93.1 \\
+\quad 9\end{array}$ & $-35 \cdot 6$ & $-29 \cdot 6$ & +57.5 \\
\hline $64 \cdot 0$ & & $-35 \cdot 2$ & $-29 \cdot 5$ & +52.3 \\
\hline 70 & $+79 \cdot 3$ & -31.6 & $-26 \cdot 4$ & +47.7 \\
\hline 80 & +66.7 & $-25 \cdot 5$ & $-21 \cdot 2$ & +41.2 \\
\hline 90 & $\begin{array}{r}+54.8 \\
\end{array}$ & $-19 \cdot 4$ & $-16 \cdot 1$ & $+35 \cdot 4$ \\
\hline 1700 & +43.6 & $-13 \cdot 2$ & -10.9 & +30.4 \\
\hline 10 & $\begin{array}{r}\mathbf{3 3} \cdot \mathbf{3} \\
+\end{array}$ & $-\mathbf{6 . 9}$ & -5.7 & +26.4 \\
\hline 20 & $\begin{array}{l}+23.8 \\
\end{array}$ & -0.6 & -0.5 & $+23 \cdot 2$ \\
\hline 30 & +15.0 & $+5 \cdot 8$ & $+4 \cdot 7$ & +20.8 \\
\hline 40 & $\begin{array}{r}7.2 \\
+\quad 7 .\end{array}$ & $+12 \cdot 2$ & $+\overline{9} .9$ & $+19 \cdot 4$ \\
\hline 50 & & $+18 \cdot 6$ & $+15 \cdot 1$ & $18 \cdot 6$ \\
\hline $\begin{array}{l}55 \cdot 3 \\
60\end{array}$ & $6 \cdot 3$ & $\begin{array}{l}+21 \cdot 9 \\
+24 \cdot 0\end{array}$ & $\begin{array}{r}+17 \cdot 7 \\
+19.5\end{array}$ & $\begin{array}{r}+18.4 \\
+17.7\end{array}$ \\
\hline 70 & $\begin{array}{l}-\quad 0.0 \\
-\quad 11.8\end{array}$ & $\begin{array}{l}+24 \cdot 0 \\
+28 \cdot 1\end{array}$ & $\begin{array}{r}+120 \\
+22.9\end{array}$ & $\begin{array}{r}+16.3 \\
+16.3\end{array}$ \\
\hline 80 & -16.5 & +31.8 & +26.2 & $+15 \cdot 3$ \\
\hline 86. & & $+34 \cdot 0$ & +28.3 & +15.0 \\
\hline 90 & $-20 \cdot 4$ & $+32 \cdot 8$ & $+27 \cdot 1$ & $12 \cdot 4$ \\
\hline 1800 & $-23 \cdot 6$ & $+29 \cdot 4$ & $+23 \cdot 8$ & $+5 \cdot 8$ \\
\hline 10 & $-\quad 25 \cdot 9$ & $+25 \cdot 7$ & +20.5 & \\
\hline 20 & $-27 \cdot 3$ & $+21 \cdot 5$ & +17.0 & \\
\hline 25 & -27.8 & $+19 \cdot 3$ & $+15 \cdot 1$ & $8 \cdot 5$ \\
\hline 30 & $-28 \cdot 0$ & $+17 \cdot 0$ & $+13 \cdot 3$ & $-11 \cdot 0$ \\
\hline 35 & $-28 \cdot 0$ & $+14 \cdot 7$ & +11.5 & -13 \\
\hline & & 42 & & \\
\hline
\end{tabular}

$\delta L=+6^{\prime \prime} \cdot 00(T+\mathrm{I})-\mathrm{Io}^{\prime \prime} \cdot 7 \mathrm{I} \sin \left(\mathrm{I}_{40} 0^{\circ} \cdot \mathrm{o} T+240^{\circ} \cdot 7\right)+0^{\prime \prime} \cdot \mathrm{I} 257 S^{\prime}+0^{\prime \prime} \cdot 5490 M$.

Pour le soleil, Mercure et Vénus, on a

$$
\begin{aligned}
& \delta \lambda_{0}=+I^{\prime \prime} \cdot 89+I^{\prime \prime} \cdot 25 T+0^{\prime \prime} \cdot 04 \mathrm{II} \Delta t, \\
& \delta \lambda_{1}=+7 \cdot 65+7 \cdot 13 T+0 \cdot 1705 \Delta t, \\
& \delta \lambda_{2}=+3 \cdot 30+2 \cdot 32 T+0 \cdot 0667 \Delta t .
\end{aligned}
$$

La table suivante donne les valeurs de $S^{\prime}, \Delta_{1} t, M$ et $\Delta t$. 


\begin{tabular}{|c|c|c|c|c|}
\hline$t$ & $\begin{array}{c}S^{\prime} \\
\mathbf{s}\end{array}$ & $\begin{array}{c}\Delta_{1} t \\
\mathrm{~s}\end{array}$ & $\begin{array}{c}M \\
\mathrm{~s}\end{array}$ & $\begin{array}{c}\Delta t \\
\mathrm{~s}\end{array}$ \\
\hline 40 & -27.9 & $+12 \cdot 3$ & +9.8 & $-15 \cdot 6$ \\
\hline 45 & $-27 \cdot 6$ & $\begin{array}{r}9.8 \\
+9.8\end{array}$ & +7.9 & -17.8 \\
\hline 50 & -27.0 & $+7 \cdot 2$ & +6.0 & -19.8 \\
\hline 55 & $-26 \cdot 2$ & +4.5 & $+4 \cdot 1$ & $-21 \cdot 7$ \\
\hline 60 & $-25 \cdot 3$ & +1.7 & $+2 \cdot 2$ & $-23 \cdot 6$ \\
\hline $64 \cdot 4$ & & -0.8 & +0.4 & -25.0 \\
\hline 65 & $-24 \cdot 1$ & -1.7 & -0.4 & -25.8 \\
\hline 70 & -22.7 & $-\overline{9} \cdot 6$ & $-7 \cdot \overline{2}$ & $-32 \cdot 3$ \\
\hline 75 & $-21 \cdot 1$ & $-17 \cdot 3$ & $-14 \cdot 0$ & -38.4 \\
\hline $76 \cdot 15$ & & $-19 \cdot 0$ & -15.5 & $-39 \cdot 7$ \\
\hline 80 & -19.4 & $-22 \cdot 4$ & $-18 \cdot 2$ & -41.8 \\
\hline 85 & $-\mathbf{1 7} \cdot \overline{4}$ & -26.7 & $-21 \cdot 8$ & $-44 \cdot 1$ \\
\hline 90 & -15.2 & $-30 \cdot 9$ & $-25 \cdot 4$ & $-46 \cdot 1$ \\
\hline 95 & -12.9 & $-34 \cdot 9$ & $-29 \cdot 0$ & -47.8 \\
\hline 96.7 & & $-36 \cdot 3$ & $-30 \cdot 1$ & $-48 \cdot 3$ \\
\hline 1900 & $-10 \cdot 4$ & $-34 \cdot 8$ & $-28 \cdot 4$ & $-45 \cdot 2$ \\
\hline 05 & -7.6 & $-32 \cdot 3$ & $-25 \cdot 7$ & -39.9 \\
\hline 10 & $-\quad 4 \cdot 6$ & $-29 \cdot 7$ & -22.9 & $-34 \cdot 3$ \\
\hline 15 & $-\quad \mathbf{1 . 3}$ & $-27 \cdot 0$ & $-20 \cdot 1$ & $-28 \cdot 3$ \\
\hline $18 \cdot 65$ & & $-24 \cdot 9$ & $-18 \cdot 2$ & $-23 \cdot 7$ \\
\hline 20 & $2 \cdot 1$ & $-25 \cdot 8$ & $-19 \cdot 1$ & 7 \\
\hline 25 & $+\quad 5 \cdot 7$ & $-29 \cdot 0$ & $-22 \cdot 4$ & $-23 \cdot 3$ \\
\hline 30 & $9 \cdot 4$ & $-32 \cdot 0$ & $-25 \cdot 8$ & $-22 \cdot 6$ \\
\hline
\end{tabular}

